Document downloaded from:

http://hdl.handle.net/10251/84850

This paper must be cited as:

Botella Mascarell, C.; Piñero Sipán, MG.; Diego Antón, MD. (2016). Multi-user interference mitigation under limited feedback requirements for WCDMA systems with base station cooperation. Telecommunication Systems. 61(3):543-557. doi:10.1007/s11235-015-0011-z.

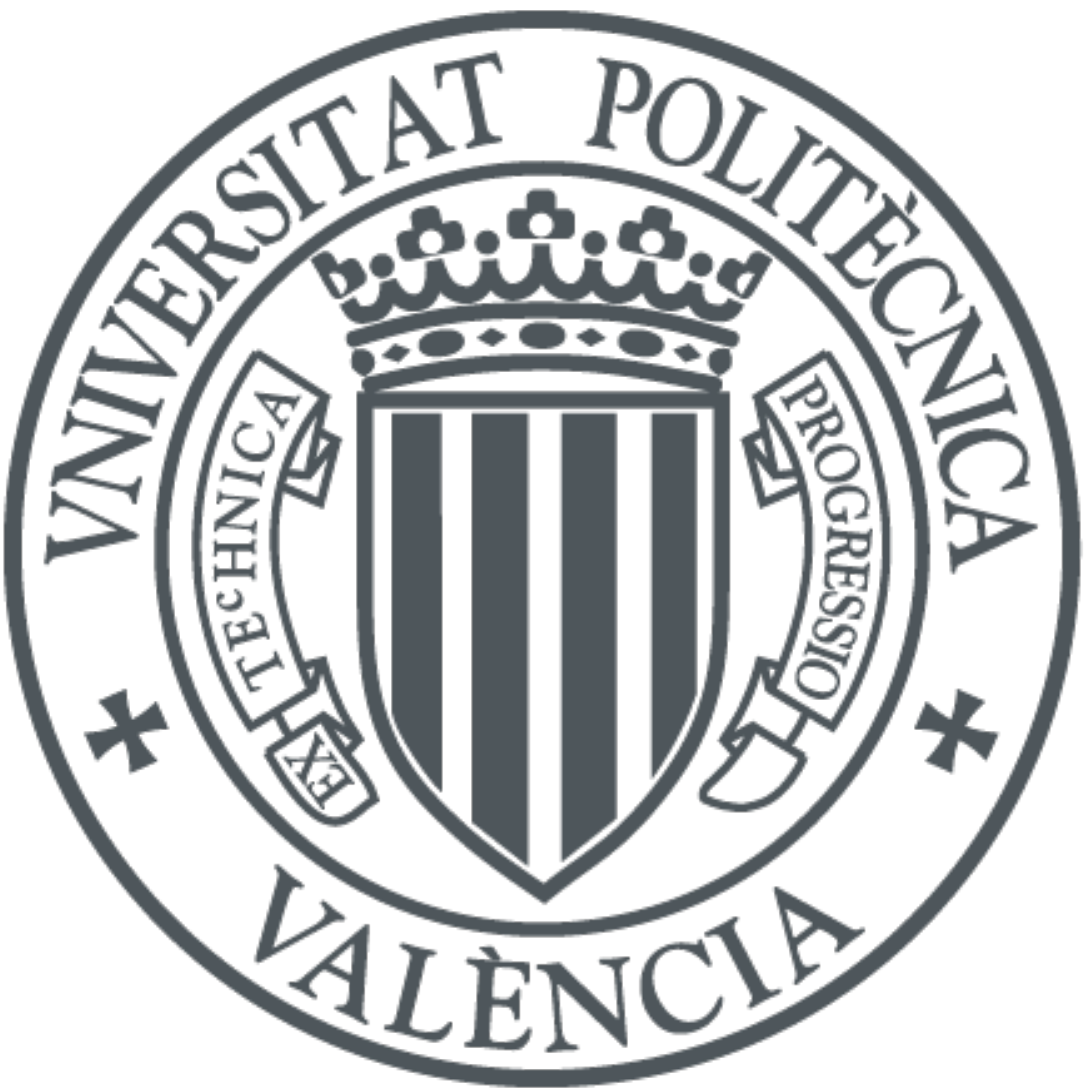

The final publication is available at

http://dx.doi.org/10.1007/s11235-015-0011-z

Copyright Springer Verlag (Germany)

Additional Information 


\title{
Multi-User Interference Mitigation Under Limited Feedback Requirements for WCDMA Systems with Base Station Cooperation
}

\author{
Carmen Botella • Gema Piñero • Maria de Diego
}

Received: date / Accepted: date

\begin{abstract}
One of the techniques that has been recently identified for dealing with multi-user interference in future communications systems is base station cooperation or joint processing. However, perfect multi-user interference cancellation with this technique demands severe synchronization requirements, perfect and global channel state information, and an increased backhaul and signaling overhead. In this paper, we consider a more realistic layout with the aim of mitigating the multi-user interference, where only local channel state information is available at the base stations. Due to synchronization inaccuracies and errors in the channel estimation, the system becomes partially asynchronous. In the downlink of WCDMA based systems, this asynchronism stands for the loss of the orthogonality of the spreading codes allocated to users and thus, for an increase in the multi-user interference level of the system. In this contribution, we propose a framework for mitigating the multi-user interference which builds in three main steps: definition of a cooperation area based on the channel characteristics, statistical modeling of the average multi-user interference power experienced by each user and a specific spreading code allocation scheme for users served with joint processing. This code allocation assigns spreading codes to users in such a way that minimum average cross-correlation between active users can be achieved. Interestingly, these steps can be
\end{abstract}

\section{Carmen Botella}

Group of Information and Communication Systems, IRTIC, Universitat de València, Valencia, Spain

E-mail: carmen.botella@uv.es

Gema Piñero · Maria de Diego

Institute of Telecommunications and Multimedia Applications (iTEAM), Universitat Politècnica de València, Spain

E-mail: \{gpinyero,mdediego\}@iteam.upv.es. performed with a limited amount of extra feedback from the user's side.

Keywords Coordinated MultiPoint - WCDMA . multi-user interference mitigation

\section{Introduction}

Base Station (BS) cooperation has been identified as one of the key techniques for mitigating inter-cell interference and achieving an ubiquitous user experience in future broadband communication systems [1]. In this approach, a group of Base Stations (BSs) acts as a single and distributed antenna array and data to a user is simultaneously transmitted from more than one BS. Therefore, potential inter-cell interference becomes useful data transmission. This technique has been also referred to in the literature as multicell cooperative processing [2], network Multiple Input Multiple Output (MIMO) [3] or distributed MIMO [4]. Ongoing work in the $3 \mathrm{GPP}$ in the framework of Long Term Evolution (LTE) Advanced renames this concept as joint transmission or processing between BSs, which falls in the umbrella of Coordinated MultiPoint (CoMP) transmission schemes [5].

Under idealized assumptions, perfect local Channel State Information ${ }^{1}$ (CSI) could be available at the transmitter side or BS. BSs could then exchange user data, local CSI and information for synchronization procedures without any constraint. From the user's point of view, and assuming a Frequency Division Duplex (FDD) setting, estimated CSI would need to be fed back to the BS. However, as the number of users and BSs increases, the major drawbacks related to BS cooperation from a practical point of view are the large

\footnotetext{
1 CSI between one BS and all the users that is serving.
} 
backhaul and signaling overhead related to the interbase information exchange and how to obtain accurate CSI at the BSs. The academic and industry communities have been actively trying to solve the question of how to design CoMP techniques to achieve the promised gains under realistic conditions and practical settings. A recent overview of the state of the art can be found in $[2]$.

The lack of efficient solutions for the problems of alleviating the backhauling load and obtaining accurate CSI at the BSs has prevented the standardization of CoMP techniques in LTE Rel-10. However, CoMP techniques have been under consideration for LTE Rel11 [6] and Rel-12 [7], since one of the foreseen solutions to cope with the huge growth in mobile broadband traffic which is expected in the short term future, is the implementation of evolved CoMP techniques in heterogeneous and ultradense networks (cooperation macrosmall cell) and the introduction of massive MIMO $[1,8$, $9]$.

Current fourth generation $(4 \mathrm{G})$ wireless standards are based on Orthogonal Frequency Division Multiple Access (OFDMA), e.g., LTE Rel-8. Due to this, most of the contributions existing in the literature regarding to different CoMP aspects assume an OFDM setting. However, High Speed Packet Access (HSPA) networks concentrate the majority of subscribers, and the major mobile broadband traffic growth is expected to come from these networks. In addition, recent improvements over HSPA have allowed to achieve competitive rates with respect to LTE, so HSPA is expected to continue evolving to perform similarly to other $4 \mathrm{G}$ technologies and even to meet IMT-Advanced requirements [10-13].

The natural question is then if CoMP-like techniques could be applied over Wideband Code Division Multiple Access (WCDMA) based networks, where the X2 interface for BS exchange is not available. To answer this question, an study item on HSDPA Multipoint Transmission was included and completed in Rel-11 [6, 14-16]. This study item focused on single point data transmission, multiflow data transmission and single frequency network data transmission. Some interesting studies can be found in [10-12,17-22]. Currently, there is a parallel evolution with respect to LTE Rel-12, and Rel-11 HSDPA multiflow is being considered as an interesting extension for heterogeneous networks (macrosmall cell multiflow) $[23,24]$. However, current version of HSDPA multiflow is limited to a two BSs transmission, while some issues related to inter-cell coordination remain unsolved.

In this paper, we consider a simplified single frequency network data transmission, where a static clus- ter ${ }^{2}$ of BSs cooperates when transmitting a data stream to a particular user. Note that clustering solutions that restrict BS cooperation to a limited number of BSs have been proposed in the literature to reduce the complexity in CoMP solutions [25-28]. In this scenario, the same carrier frequency and the same scrambling code are used in different sectors. Within the cluster, the signal arriving at the user experiences different propagation delays from each BS. Following the joint processing approach, the user tries to coherently combine the data coming from the different BSs. At this point, most of the previous works simplify the system model by assuming synchronous reception at the user, implying perfect and instantaneous estimation of all the channels in the system, which seems rather unrealistic. In [29], a system model with timing-advance mechanisms is developed in order to ensure that each user receives a coherent sum of its desired signals, whereas the interference term remains asynchronous. Analysis of imperfect timing-advance scenarios is also carried out. This work is extended in [30], whereas [31] presents an analytical interference analysis for the downlink of asynchronous OFDM multicellular networks. In [32], the authors focus on the estimation and correction of the carrier frequency offsets that may occur in multicellular networks allowing cooperation.

In this work, we assume that transmissions from BSs within the cluster cannot be perfectly synchronized and then each user in the cluster receives both the desired and interference signals from the multiple BSs in an asynchronous manner. In current WCDMAbased systems, Orthogonal Variable Spreading Factor (OVSF) or Walsh-Hadamard codes are employed to preserve the orthogonality between different users. In a typical uplink wireless environment with multipath, these spreading codes lose their orthogonality due to the time-shifted versions that arrive at the receiver. In traditional systems, this intra-cell interference can be removed using different equalization techniques. In a similar way, in the downlink channel of a system transmitting with BS cooperation, even if a frequency flat channel is considered, the asynchronous nature of the signals arriving at each receiver would introduce Multiple Access Interference (MAI). Traditionally, the loss of orthogonality in CDMA downlink is modeled in the analysis and simulation of CDMA systems by means of the time average value of the orthogonality factor. ${ }^{3}$ Some authors, [33-35], have worked in a complete characterization of this parameter. However, attending to

\footnotetext{
2 The cluster remains fixed in time.

3 The orthogonality factor is defined as the instantaneous fraction of received downlink power converted by multipath into interference.
} 
the special features related to BS cooperation, a further analysis is required. Note that some prior work can be found in [36].

In this paper, a statistical system model is developed to characterize the interference contributions received by a user in the framework of a WCDMA system with BS cooperation. This interference modeling is carried out taking into account the correlation properties of the time-shifted spreading codes. Moreover, it will be shown that only limited feedback information from each user and local CSI at each BS are required. Therefore, from a practical point of view, the backhaul and feedback signaling overhead are not remarkably increased. Interestingly, this characterization points out the relation between the Mean Squared Value (MSV) of the correlation terms of the spreading codes and the interference contributions experienced by the users.

In systems with BS cooperation, additional mechanisms, such as resource allocation techniques [37], may be required to deal with the interference contributions that cannot be fully mitigated using spatial processing. In this paper, a multi-base spreading code allocation scheme is specifically proposed for systems with BS cooperation, where spreading codes are assigned to users in such a way that minimum average cross-correlation between active users can be achieved. This scheme can be implemented in a distributed manner, decreasing the need of additional infrastructure modifications. In addition to this scheme, a modified version of the joint power control and beamforming algorithm from [38] and [39] is performed by the BSs within the cluster. Finally, another aim of this contribution is to give some insights into the question of until which extent is beneficial to cooperate in a partially asynchronous WCDMA system. We assume that only local CSI is available at the BSs.

To summarize, the main contribution of this paper is the development of a framework for multi-user interference mitigation based on the following steps:

- Definition of a cooperation area $^{4}$ inside the cluster of BSs based on the maximum delay of the composite channel. Section 3 states the benefits of this solution with respect to more conventional channel gain-based solutions.

- Analytical characterization of the multi-user interference for the downlink of a static cluster with base station cooperation in a WCDMA framework.

- Derivation of a distributed multi-base spreading code allocation scheme to minimize the multi-user interference experienced by the users.

\footnotetext{
4 Area where BS cooperation is actually performed.
}

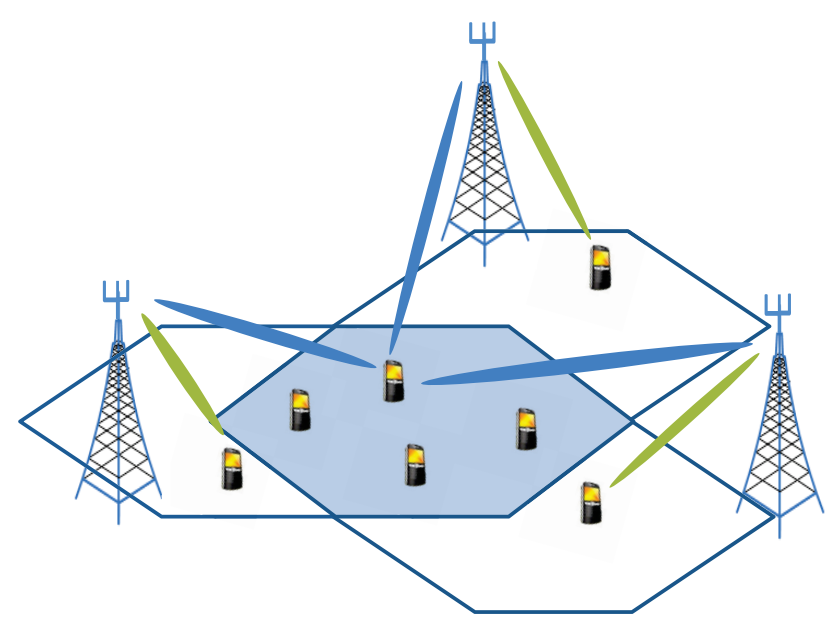

Fig. 1 Cooperation cluster with $K=3$ BSs. Note that joint processing or base station cooperation is only allowed in the shadowed area

The paper is organized as follows. In Section 2, the system model for BS cooperation in a WCDMA multicell system is presented, whereas the statistical interference modeling and the definition of the cooperation area are proposed in Section 3. Section 4 introduces the multi-base spreading code allocation scheme, and Section 5 describes the simulation assumptions and numerical results. Finally, conclusions are drawn in Section 6 . The following notation is used throughout the paper: boldface upper-case letters denote matrices, boldface lower-case letters denote vectors and italics denote scalars. Superscripts $(\cdot)^{T},(\cdot)^{*}$ and $(\cdot)^{H}$ denote the transpose, the conjugate and the conjugate transpose operations, respectively. $E[\cdot]$ denotes mathematical expectation and $\|\cdot\|$ stands for the 2-norm.

\section{System Model}

Consider a cluster of $K \mathrm{BSs}$, each one equipped with $N_{a}$ antennas, and $M$ users that can be equipped with $N_{r}$ antennas, $N_{r} \geq 1$. With joint processing or cooperation between BSs, data to a user is simultaneously transmitted from more than one BS, see Fig. 1.

Regarding the particular information signals, users are served and channelized through OVSF codes $c_{m}(t)$. All possible codes share the same chip period, $T_{c}$, but may have different spreading factor, $N$. Second step of spreading procedure or scrambling operation is not considered here since it is assumed that BSs within the cluster share the scrambling code in order to achieve a reuse factor of 1 . Therefore, the OVSF code for the $m$ th user, $m=1, \ldots, M$, is written as:

$c_{m}(t)=\sum_{n_{c}=0}^{N-1} c_{m}\left[n_{c}\right] p\left(t-n_{c} T_{c}\right)$ 
where $c_{m}\left[n_{c}\right]$ is the spreading code and $p(t)$ is the impulse response of the Nyquist pulse shaping filter. The information signal $u_{m}(t)$ transmitted to the $m$ th user is linearly modulated:

$u_{m}(t)=\sum_{j=-\infty}^{\infty} a_{m}[j] c_{m}(t-j T)$.

Here, $T=N T_{c}$ denotes the symbol period and $a_{m}[j]$ is the data symbol. Without loss of generality, we assume that $E\left[\left|u_{m}(t)\right|^{2}\right]=1$.

Assume that only local CSI is available at each BS. Therefore, even if timing-advanced mechanisms are applied to ensure a synchronous reception at the user, due to channel estimation errors and synchronization inaccuracies, the cluster becomes partially asynchronous. The channel between the $k$ th BS and the $m$ th user is then modeled as a MIMO multipath channel:

$\mathbf{H}_{m, k}(t)=\sum_{l=1}^{L} \mathbf{H}_{m, k, l} \delta\left(t-\tau_{m, k, l}\right)$,

where $\mathbf{H}_{m, k, l}$ is the $\left[N_{r} \times N_{a}\right]$ matrix of the $l$ th multipath components, $\tau_{m, k, l}$ is the relevant propagation time delay common to the $l$ th multipath components of the $k$ th BS - $m$ th user link ${ }^{5}$, and $L$ is the maximum number of propagation paths of the same link. Elements of matrices $\mathbf{H}_{m, k, l}$, denoted by $h_{m, k, l}^{(i, j)}$, represent the fading amplitude between transmitter antenna $j$ and receiver antenna $i$, and are modeled as independent and identically distributed (i.i.d.) circularly symmetric complex Gaussian variables, with zero mean and unit variance, $\mathcal{C N}(0,1)$. Finally, as $\mathbf{H}_{m, k}(t)$ is dependent only on $t$, channel fadings are considered time-invariant within a block of transmitted data. Notice that propagation delays $\tau_{m, k, l}$ are considered arbitrary at this point and they are not required to be multiples of $T_{c}$. Assume that for all the $(m, k)$ multipath channels, propagation delays are ordered $\left(\tau_{m, k, 1}<\tau_{m, k, 2}<\cdots<\tau_{m, k, L}\right)$ and they are also resolvable $\left(\tau_{m, k, l+1}-\tau_{m, k, l}>T_{c}\right)$.

Base station cooperation requires user data to be available at each BS in the cluster. Denoting by $\mathbf{w}_{i k}$ the $\left[N_{a} \times 1\right]$ precoding or beamforming vector of the $k$ th BS for the $i$ th user, the signal transmitted from the $k$ th BS can be written as:

$\mathbf{x}_{k}(t)=\sum_{i=1}^{M} \sqrt{P_{i, k}} \mathbf{w}_{i, k} u_{i}(t)$,

5 Since the system model considers linear arrays of $N_{a}$ and $N_{r}$ antennas with half of the wavelength separation colocated at the BS and user device, respectively, we can assume that the different angles of arrival of the received signal are common to all the antennas [40], and so are the propagation delays $\tau_{m, k, l}$. where $P_{i, k}\left\|\mathbf{w}_{i, k}\right\|^{2}$ is the downlink power allocated to the $i$ th user. Considering that signals $u_{i}(t)$ are uncorrelated for $i \neq j$, and assuming that $P$ is the maximum transmitted power per BS, the transmitted signal is such that $E\left[\left\|\mathbf{x}_{k}\right\|^{2}\right] \leq P$, that is, $\sum_{i=1}^{M} P_{i, k}\left\|\mathbf{w}_{i, k}\right\|^{2} \leq$ $P, \forall k$.

Taking into account the multipath channel model (3), the received signal vector of dimensions $\left[N_{r} \times 1\right]$ at the $m$ th user is given by the sum of all the BSs contributions:

$$
\begin{aligned}
& \mathbf{y}_{m}^{r}(t)=\sum_{k=1}^{K} \sum_{l=1}^{L} \mathbf{H}_{m, k, l} \mathbf{x}_{k}\left(t-\tau_{m, k, l}\right)+\mathbf{z}_{m}^{r}(t) \\
& =\sum_{i=1}^{M} \sum_{k=1}^{K} \sqrt{P_{i, k}} \sum_{l=1}^{L} \mathbf{H}_{m, k, l} \mathbf{w}_{i, k} u_{i}\left(t-\tau_{m, k, l}\right)+\mathbf{z}_{m}^{r}(t),
\end{aligned}
$$

where $\mathbf{z}_{m}^{r}(t)$ is the $\left[N_{r} \times 1\right]$ thermal noise vector at the $m$ th receiver.

We assume that only local CSI and user's data are available at each BS in the cluster. Due to this, multiuser interference within the cluster cannot be perfectly canceled through joint processing. In this case, it is convenient to express the received signal (5) considering separately contributions from the desired signal, $u_{m}(t)$, the multi-user interference signal, $u_{i}(t)$ with $i \neq m$, and the thermal noise:

$$
\begin{aligned}
\mathbf{y}_{m}^{r}(t) & =\sum_{k=1}^{K} \sqrt{P_{m, k}} \sum_{l=1}^{L} \mathbf{H}_{m, k, l} \mathbf{w}_{m, k} u_{m}\left(t-\tau_{m, k, l}\right) \\
& +\sum_{\substack{i=1 \\
i \neq m}}^{M} \sum_{k=1}^{K} \sqrt{P_{i, k}} \sum_{l=1}^{L} \mathbf{H}_{m, k, l} \mathbf{w}_{i, k} u_{i}\left(t-\tau_{m, k, l}\right) \\
& +\mathbf{z}_{m}^{r}(t) .
\end{aligned}
$$

Once received, baseband signal $\mathbf{y}_{m}^{r}(t)$ enters a $2 \mathrm{D}$ RAKE receiver in order to exploit diversity. Let us name $\mathbf{r}_{m, q}$ the $\left[1 \times N_{r}\right]$ vector associated to the $q$ th finger of the $m$ th user RAKE. $\mathbf{r}_{m, q}$ is usually designed to compensate the channel fading of the time-aligned signal in such a way that combining all the fingers' outputs enhances the received Signal-to-Noise Ratio (SNR) [41]. The output signal at $t=d T$ in the $q$ th finger of the $m$ th user after correlation with the OVSF $\operatorname{code} c_{m}(t)$ is given by [42]:

$$
\begin{aligned}
& y_{m, q}[d]=\mathbf{r}_{m, q} \cdot \\
& {\left[\sum_{k=1}^{K} \sqrt{P_{m, k}} \sum_{l=1}^{L} \mathbf{H}_{m, k, l} \mathbf{w}_{m, k} \rho_{m m}\left(\beta_{m, q}-\tau_{m, k, l}\right) a_{m}[d](9)\right.} \\
& +\sum_{\substack{i=1 k=1 \\
i \neq m}}^{M} \sqrt{P_{i, k}} \sum_{l=1}^{L} \mathbf{H}_{m, k, l} \mathbf{w}_{i, k} \rho_{m i}\left(\beta_{m, q}-\tau_{m, k, l}\right) a_{i}[d](10) \\
& \left.+\mathbf{z}_{m, q}[d]\right],
\end{aligned}
$$


where

$\rho_{m i}\left(\beta_{m, q}-\tau_{m, k, l}\right)=$

$=\frac{1}{T} \int_{d T}^{(d+1) T} c_{i}\left(t+\left(\beta_{m, q}-\tau_{m, k, l}\right)\right) c_{m}^{*}(t-d T) d t$,

is the correlation of orthogonal codes $m$ and $i$ and $\beta_{m, q}$ is the delay associated to the $q$ th correlator of user $m$. $\rho_{m m}(\cdot)$ in $(9)$ is the autocorrelation of code $m$ with the same expression as in (12) but for $i=m$, and

$\mathbf{z}_{m, q}[d]=\frac{1}{T} \int_{d T}^{(d+1) T} \mathbf{z}_{m}^{0}\left(t+\beta_{m, q}\right) c_{m}^{*}(t-d T) d t$,

is the noise term. Notice that when $t=d T$, equation (9) contains the desired symbol $a_{m}[d]$.

From this point on and for the sake of simplicity, we will consider $N_{r}=1$ antennas at the individual receivers. Therefore, fingers' vectors become scalar gains $r_{m, q}$, and fading matrices $\mathbf{H}_{m, k, l}$ turn into $\left[1 \times N_{a}\right]$ fading vectors $\mathbf{h}_{m, k, l}$, and (9)-(11) can be expressed as follows:

$$
\begin{aligned}
& y_{m, q}[d]=r_{m, q} . \\
& {\left[\sum_{k=1}^{K} \sqrt{P_{m, k}} \sum_{l=1}^{L} \mathbf{h}_{m, k, l} \mathbf{w}_{m, k} \rho_{m m}\left(\beta_{m, q}-\tau_{m, k, l}\right) a_{m}[d](13)\right.} \\
& +\sum_{\substack{i=1 k=1 \\
i \neq m}}^{M} \sqrt{P_{i, k}} \sum_{l=1}^{L} \mathbf{h}_{m, k, l} \mathbf{w}_{i, k} \rho_{m i}\left(\beta_{m, q}-\tau_{m, k, l}\right) a_{i}[d] \\
& \left.+z_{m, q}[d]\right] .
\end{aligned}
$$

As the scope of this work is to characterize and mitigate the Multi-User Interference (MUI) due to asynchronous reception in the downlink of a WCDMA cluster of BSs, it seems convenient to make some model assumptions that simplify the notation of Eq. (13)-(15) but will not alter the MUI expression of the model:

A.1. The number of fingers available, $Q$, is equal to the number of BSs in the cluster, $Q=K$. This particular choice of $Q$ is the minimum value that allows for the exploitation of the spatial diversity of the $K$ BSs' signals.

A.2. The highest gain path of each BS-user channel is its first arriving path $\mathbf{h}_{m, k, 1}$, that is, $\mathbf{h}_{m, k, 1}$ is such that:

$$
\left\|\mathbf{h}_{m, k, 1}\right\|=\max _{l}\left\|\mathbf{h}_{m, k, l}\right\|, \quad k=1, \ldots, K .
$$

A.3. The $q$ th correlator is synchronized with the highest gain path of the $k=q$ BS-user propagation channel:

$$
\beta_{m, q}=\tau_{m, q, 1}, q=1, \ldots, K
$$

A.4. The value of $r_{m, q}$ is designed as an Equal Ratio Combining (ERC). Considering assumption A.1, the value of the $q$ th finger coefficient $r_{m, q}$ can be expressed as:

$$
r_{m, q}=\frac{\left(\mathbf{h}_{m, q, 1} \mathbf{w}_{m, q}\right)^{*}}{\left\|\mathbf{h}_{m, q, 1} \mathbf{w}_{m, q}\right\|^{2}}, \text { for } q=1, \ldots, K
$$

A.5. The symbol duration $T$ is considered to be longer than the maximum delay spread across the $K \cdot M$ propagation channels, and hence, the Inter-Symbol Interference (ISI) term can be ignored. That is, the cluster is able to use timing-advance mechanisms to ensure a symbol-time synchronous transmission and reception.

Following the above assumptions, and taking into account that the $Q$ correlator outputs are summed up after the despreading process, the $m$ th user receiver output can be expressed as:

$y_{m}[d]=\sum_{q=1}^{K} y_{m, q}[d]=y_{m}^{\mathrm{SOI}}[d]+y_{m}^{\mathrm{IFI}}[d]+y_{m}^{\mathrm{MUI}}[d]+y_{m}^{\mathrm{Z}}[d]$

where

$y_{m}^{\mathrm{SOI}}[d]=\left(\sum_{q=1}^{K} \sqrt{P_{m, q}}\right) a_{m}[d]$

is the Signal of Interest (SOI) term obtained from (13) and (18) for $k=q$ and $l=1$.

The Inter-Finger Interference (IFI) term is formed by all the terms in (13) and (18) containing symbols $a_{m}[d]$ except those of (19):

$$
\begin{aligned}
& y_{m}^{\mathrm{IFI}}[d]= \\
& =\sum_{q=1}^{K} r_{m, q}\left[\sqrt{P_{m, q}} \sum_{l=2}^{L} \mathbf{h}_{m, q, l} \mathbf{w}_{m, q} \rho_{m m}\left(\beta_{m, q}-\tau_{m, q, l}\right)\right. \\
& \left.+\sum_{\substack{k=1 \\
k \neq q}}^{K} \sqrt{P_{m, k}} \sum_{l=1}^{L} \mathbf{h}_{m, k, l} \mathbf{w}_{m, k} \rho_{m m}\left(\beta_{m, q}-\tau_{m, k, l}\right)\right] a_{m}[d] .
\end{aligned}
$$

Notice that the first term inside the bracket corresponds to the contributions arriving from the BS synchronized with the $q$ th finger, $k=q$, but whose delays are not synchronized with the finger delay $\left(\beta_{m, q} \neq \tau_{m, k, l}\right)$. 
The MUI term can be expressed from (14) and (18) as:

$$
\begin{aligned}
& y_{m}^{\mathrm{MUI}}[d]= \\
& =\sum_{q=1}^{K} r_{m, q}\left[\sum_{\substack{i=1 \\
i \neq m}}^{M} \sqrt{P_{i, q}} \sum_{l=2}^{L} \mathbf{h}_{m, q, l} \mathbf{w}_{i, q} \rho_{m i}\left(\beta_{m, q}-\tau_{m, q, l}\right)\right. \\
& \left.+\sum_{\substack{i=1 \\
i \neq m}}^{M} \sum_{\substack{k=1 \\
k \neq q}}^{K} \sqrt{P_{i, k}} \sum_{l=1}^{L} \mathbf{h}_{m, k, l} \mathbf{w}_{i, k} \rho_{m i}\left(\beta_{m, q}-\tau_{m, k, l}\right)\right] a_{i}[d],
\end{aligned}
$$

where the term $k=q, l=1$, has been omitted since $\rho_{m i}\left(\beta_{m, q}-\tau_{m, q, 1}\right)=\rho_{m i}(0)=0$.

Finally, the noise term is expressed as:

$y_{m}^{\mathrm{Z}}[d]=\sum_{q=1}^{K} r_{m, q} z_{m, q}[d]$.

As it can be seen from the IFI and MUI expressions, (20)-(21), both terms depend on the correlation function: the autocorrelation term $\rho_{m m}(\cdot)$ in the case of the IFI and the cross-correlation one, $\rho_{m i}(\cdot)$, in the case of the MUI. In order to mitigate the interference suffered by each user, IFI and MUI terms (20)-(21) need to be considered. Regarding the IFI, or self-interference suppression, it has been widely studied and good performance solutions as Minimum Mean Square Error (MMSE) or generalized RAKE receivers [43,44] could be used. However, the MUI is completely unknown at the receiver and cannot be estimated as it can be done in the uplink channel. Therefore, the following Section focuses on the MUI term (21), and derives a closedform expression for the MUI contribution experienced by each user in the cluster.

\section{Modeling of the Multi-User Interference}

In this Section, the objective is to model the crosscorrelation terms $\rho_{m i}(\cdot)$ in the MUI expression in (21), which will allow the derivation of an interference mitigation strategy at the transmitter side. More precisely, this strategy will follow three steps: the definition of a cooperation area inside the cluster, the statistical modeling of cross-correlation terms and the use of a multibase spreading code allocation scheme common to the multiple BSs transmitting to each user.

The average MUI power received by the $m$ th user in the cluster is given by:

$$
P_{m}^{\mathrm{MUI}}=E\left[\left|y_{m}^{\mathrm{MUI}}[d]\right|^{2}\right],
$$

where $y_{m}^{\mathrm{MUI}}[d]$ is expressed in (21). Before evaluating this interference power, we will discuss how the expectation operator is affecting each random variable involved in (21).

\section{- Correlation of data symbols:}

Data symbols from different users are assumed to be uncorrelated, $E\left[a_{i}[d] a_{j}^{*}[d]\right]=0, \forall i \neq j$, so crossterms of the summation over $M$ in (21) will be zero. Regarding the case $i=j$, after assuming in (2) that the transmitted information signal fulfilled the condition $E\left[\left|u_{m}(t)\right|^{2}\right]=1$, the average energy of linear modulated symbols is $E\left[\left|a_{i}[d]\right|^{2}\right]=1$.

- Correlation of multi-path channel gains:

The expectation in (21) will affect the complex scalars resulting from $\left(\mathbf{h}_{m, k, l} \mathbf{w}_{i, k}\right)$ inner products as:

$\mathbf{w}_{i, j}^{H} E\left[\mathbf{h}_{m, j, p}^{H} \mathbf{h}_{m, k, l}\right] \mathbf{w}_{i, k}$,

$j, k=1, \ldots, K, p, l=1, \ldots, L$.

Considering a multi-cell scenario, channel gains from different BSs are uncorrelated, so the terms $j \neq k$ are null. Regarding the cross-correlation between the channel gains of the same BS, $j=k$, channel taps occurring at different delays, $p \neq l$, correspond to paths originated from different scatterers in the geometrically-based stochastic model proposed by 3 GPP in [40]. From the model proposed in [40] and its spatial-temporal correlation stated in [45], only the channel taps originated by the same scatterer are correlated, what is called a cluster [40, 45]. Therefore, the expectation in (24) will be zero for taps originated from different clusters of scatterers, $p \neq l$, so (24) becomes:

$$
\begin{aligned}
& \mathbf{w}_{i, j}^{H} E\left[\mathbf{h}_{m, j, p}^{H} \mathbf{h}_{m, k, l}\right] \mathbf{w}_{i, k}= \\
& =\left\{\begin{array}{cc}
\mathbf{w}_{i, k}^{H} E\left[\mathbf{h}_{m, k, l}^{H} \mathbf{h}_{m, k, l}\right] \mathbf{w}_{i, k}, j=k, p=l, \\
0, & \text { otherwise. }
\end{array}\right.
\end{aligned}
$$

Following the above correlation properties, the average MUI power (23) can be expressed as:

$$
\begin{aligned}
& P_{m}^{\mathrm{MUI}}= \sum_{q=1}^{K}\left[\sum_{\substack{i=1 \\
i \neq m}}^{M} P_{i, q} \sum_{l=2}^{L} \mathbf{w}_{i, q}^{H} E\left[\mathbf{h}_{m, q, l}^{H} \mathbf{h}_{m, q, l}\right] .\right. \\
& \mathbf{w}_{i, q} \rho_{m i}^{2}\left(\beta_{m, q}-\tau_{m, q, l}\right)+ \\
& \sum_{\substack{i=1 \\
i \neq m}}^{M} \sum_{\substack{k=1 \\
k \neq q}}^{K} P_{i, k} \sum_{l=1}^{L} \mathbf{w}_{i, k}^{H} E\left[\mathbf{h}_{m, k, l}^{H} \mathbf{h}_{m, k, l}\right] . \\
&\left.\mathbf{w}_{i, k} \rho_{m i}^{2}\left(\beta_{m, q}-\tau_{m, k, l}\right)\right]
\end{aligned}
$$


where we have applied $\left|r_{m, q}\right|^{2}=1$, see (17). Let us re-arrange the sum terms of (26) using the assumption stated in A.3, that is, $\beta_{m, q}=\tau_{m, q, 1}, q=1, \ldots, K$ : $P_{m}^{\mathrm{MUI}}=$

$$
\begin{gathered}
=\sum_{\substack { q=1 \\
\begin{subarray}{c}{i=1 \\
i \neq m{ q = 1 \\
\begin{subarray} { c } { i = 1 \\
i \neq m } }\end{subarray}}^{K}\left[\sum_{\substack{k=1 \\
k \neq q}}^{K} P_{i, k} \mathbf{w}_{i, k}^{H} E\left[\mathbf{h}_{m, k, 1}^{H} \mathbf{h}_{m, k, 1}\right] \cdot\right. \\
\mathbf{w}_{i, k} \rho_{m i}^{2}\left(\tau_{m, q, 1}-\tau_{m, k, 1}\right)+ \\
\sum_{k=1}^{K} P_{i, k} \sum_{l=2}^{L} \mathbf{w}_{i, k}^{H} E\left[\mathbf{h}_{m, k, l}^{H} \mathbf{h}_{m, k, l}\right] \\
\left.\mathbf{w}_{i, k} \rho_{m i}^{2}\left(\tau_{m, q, 1}-\tau_{m, k, l}\right)\right]
\end{gathered}
$$

The first term (27) is the average MUI power received through the first path of the BSs different from the one whose RAKE finger is synchronized to, $\tau_{m, k, 1}, \forall k \neq q$, whereas the second term, (28), includes the MUI arriving from paths $l=2, \ldots, L$. The question now is how the cluster can deal with the mitigation of the average MUI power. From (27)-(28) we see that the task is dual: on the one hand, through an accurate joint design of the transmitted power $P_{i, k}$ and beamformers $\mathbf{w}_{i, k}$, and on the other hand, reducing the cross-correlation term $\rho_{m i}\left(\tau_{m, q, 1}-\tau_{m, k, l}\right)$. In the case of OVSF or Walsh codes, if perfect and instantaneous CSI were available at the BSs, it would be possible to obtain the actual correlation values $\rho_{m i}\left(\tau_{m, q, 1}-\tau_{m, k, l}\right)$. Moreover, this knowledge could be used to mitigate the average MUI power in the cluster. From a practical point of view, it could be possible to substitute these exact values by an estimate of the MUI correlation terms, $\rho_{m i}\left(\tau_{m, q, 1}-\tau_{m, k, l}\right)$, given a certain model of the relative delays $\left(\tau_{m, q, 1}-\tau_{m, k, l}\right)$.

Cross-correlation terms $\rho_{m i}\left(\tau_{m, q, 1}-\tau_{m, k, l}\right)$ depend on how the delays or Time of Arrival (ToA) of the different paths are characterized for every single channel BSuser $(q=k, l \neq 1)$, but they also depend on the difference between ToAs from different BSs $(q \neq k, \forall l)$. Fig. 1 shows the cooperation cluster with $K=3$ BSs where only users located in the shadowed area are served using joint processing. Throughout the paper, the term cluster is used for the set of BSs engaged in cooperation and for the shadowed area where cooperation takes place. The composite channel seen by one particular user is the combination of three individual channels, one per BS in the cluster. Fig. 2 shows an example of the Power Delay Profile (PDP) of each individual channel together with the resulting composite channel considering the urban micro model of 3GPP [40]. Fig. 2 also indicates the ToA of the first path for each individual link, $\tau_{m, k, 1}$

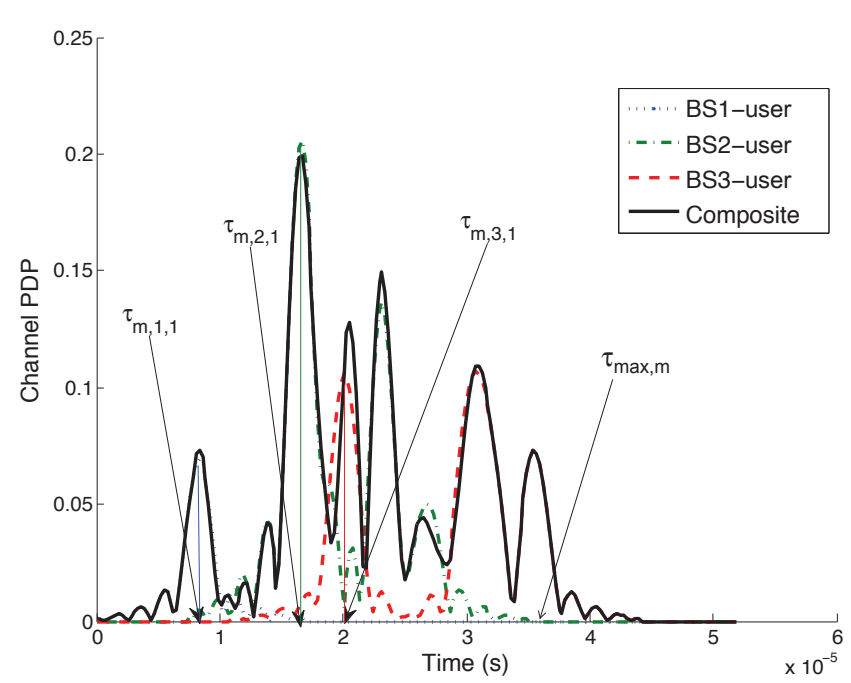

Fig. 2 Example of a composite PDP in a cooperation cluster with $K=3 \mathrm{BSs}$

for $k=1,2,3$. For the sake of simplicity, the numbering of the delays is taken correlative to their value, that is, $\tau_{m, 1,1}$ is the minimum and $\tau_{m, 3,1}$ is the maximum, so the numbering of the BSs is not fixed in our cluster setting, but it depends on the ToAs within the composite channel. Moreover, we assume that BS1, that is, the one related to $\tau_{m, 1,1}$, is the serving BS for user $m$ for feedback purposes. This assumption holds for each user in the cluster and for the remaining of the paper.

In theory, all users located in the shadowed area of Fig. 1 will be served using joint processing. However, since the composite channel is a combination of three individual channels, and users are synchronized to their serving BS, the spreading codes received at the user from different BSs lose their orthogonality and the average MUI power in the cluster is increased. To mitigate this effect, we will define a cooperation area based on the distribution of $\tau_{m, k, 1}, \forall k$, the statistical model of BS-user channels, and the resulting statistical model of the maximum delay of the composite channel denoted as $\tau_{\max , m}$ in Fig. 2. We will show how the distribution of the channels' ToA, $\tau_{m, k, 1}$, determines the maximum delay of the composite channel, $\tau_{\max , m}$, and affects the average MUI power experienced by the users.

\subsection{Definition of the cooperation area based on the} maximum delay of the composite channel

We define the cooperation area as the area within the cluster where the maximum delay of the composite channel does not exceed a predefined threshold $\tau_{\max }$. As mentioned above, the aim is to mitigate the impact of non-orthogonal spreading codes. The predefined threshold $\tau_{\max }$ depends on the spreading factor $N$ and on 
the channel distribution and it could be dynamically adapted to shrink or extend the cooperation area according to the system requirements.

Actually, the definition of a cooperation area can be seen as forming a subcluster, which would be a networkcentric solution, since the decision is taken at the network level. Other cooperation area definitions can be found for example in [46], where a pathloss-based cooperation area is proposed. Opposite to network-centric, we find the user-centric clustering or subclustering techniques, as for example in $[38,47,48]$. In $[38,47]$, a channel gain-based subclustering technique is proposed, where user-centric overlapping subclusters are defined for each user. The problem of this approach is the difficulty of grouping users sharing the same subcluster, which results in a small penetration rate, as it has been pointed out in [49]. This effect is avoided by using the definition of the cooperation area as proposed in this paper, which, in contrast to [46], also takes into account the randomness of the channel. Note that when more than one cluster are considered, advanced solutions such as the proposed in [1] will be needed to deal with the intercluster interference.

Let us define the time of arrival difference (ToAD) between $\mathrm{BS}^{6}$ and the remaining $\mathrm{BSs}$ in the cluster for user $m$ as $\Delta T_{m k}=\tau_{m, k, 1}-\tau_{m, 1,1}$, with $k=2, \ldots, K$. Following the approach presented in [50] for OFDM systems, the ToAD can be approximated as:

$\Delta T_{m k}=\tau_{m, k, 1}-\tau_{m, 1,1} \approx \frac{d_{m k}-d_{m 1}}{c}, \quad k=2, \ldots, K$,

where $d_{m 1}$ and $d_{m k}$ are the distance between the $m$ th user location and BS1 and between the $m$ th user location and BS $k$, respectively, and constant $c$ is the speed of light. Note that (29) measures the line of sight time difference, which is an approximation of the actual time difference between signals.

Let us now define the maximum delay of the composite channel of the $m$ th user as:

$\tau_{\max , m}=\max _{k}\left\{\Delta T_{m k}+\tau_{m, k, L}\right\}$,

where $\tau_{m, k, L}$ denotes the ToA of the last path, $L$, for any BS $k$. For example, in Fig. $2, \tau_{\max , m}$ corresponds to the last path of BS3, that is, $\tau_{\max , m}=\Delta T_{m 3}+\tau_{m, 3, L}$.

We define the Cooperation Area (CA) as the area within the cluster where the maximum delay of any user's composite channel is such that:

$\max _{m}\left\{\tau_{\max , m}\right\} \leq \tau_{\max }, \quad \forall m$,

6 As remarked in the previous Section, the numbering of the BSs is not fixed, but it depends on the composite channel state of a given user: BS1, in addition of being the serving BS for user $m$, is that whose $\tau_{m, k, 1}$ is the minimum.

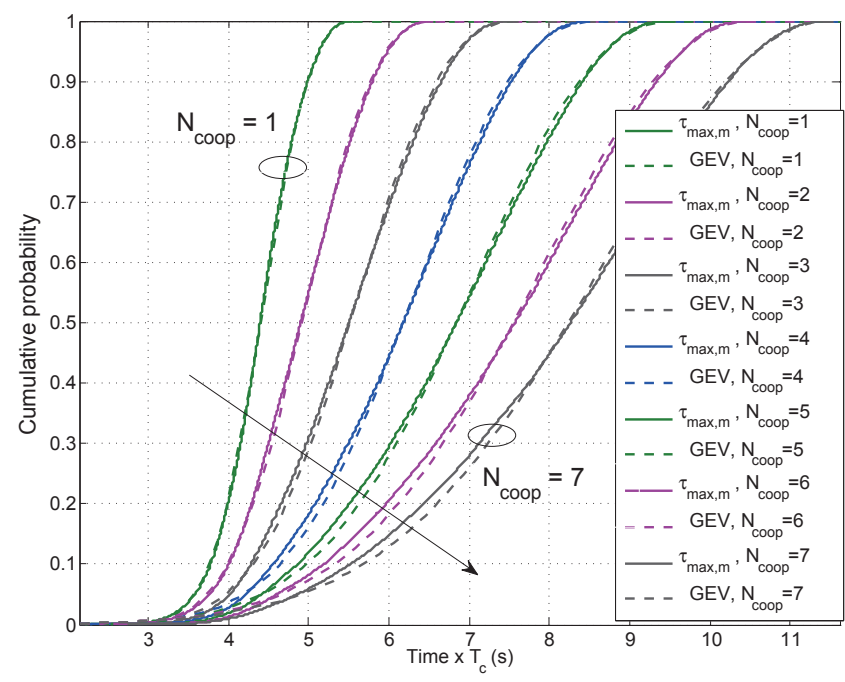

Fig. 3 CDFs of the maximum delay of the composite channel, $\tau_{\max , m}$ for different values of $N_{\text {coop }}$, a cluster of $3 \mathrm{BSs}$ and channels modeled as urban micro [40]. Their corresponding Generalized Extreme Value (GEV) approximations are plotted in dashed line

where variable $m$ stands for all the possible user locations in the cluster area and $\tau_{\max }$ is the maximum delay established by the network. Once this area is defined, only the users located in this area will be served with joint processing.

In the following, we will state a procedure to relate the CA defined in (31) to the maximum ToAD in (29) measured in number of chips, which will give us a tool to define the CA inside the cluster and to determine which users will be served through joint processing. Assuming a certain statistical model of the channel's last delay, $\tau_{m, k, L}, \forall m, k$, and a certain distribution of the ToADs, $\Delta T_{m k}, \forall k$, the statistical behavior of $\tau_{\max , m}$ in (30) can be stated. We will explain it with an example.

Consider a uniform distribution of user locations within the cluster area of Fig. 1, such that their ToADs can be assumed uniformly distributed within $\Delta T_{m k} \sim$ $\mathcal{U}\left[0, N_{\text {coop }} T_{c}\right]$, where $T_{c}=1 / 3.84 \cdot 10^{-6} \mathrm{~s}$ and $N_{\text {coop }}$ is a positive integer, $N_{\text {coop }}>0$. Assume that the channels can be characterized as urban micro [40], whose PDP consists of 6 distinct paths following a uniform distribution $\tau_{m, k, l} \sim \mathcal{U}[0,1.2 \mu s]$. In order to obtain the Cumulative Density Function (CDF) of $\tau_{\max , m}$ in (30) for different values of $N_{\text {coop }}$, we have carried out 30000 runs of Monte-Carlo simulations. The resulting CDFs and their corresponding Generalized Extreme Value (GEV) approximations are shown in Fig. 3. Once the CDFs are obtained, we can define the probability of cooperation, $P_{\text {coop }}$, based on (31), as:

$P_{\text {coop }}=\mathcal{P}\left\{\tau_{\text {max }, m} \leq \tau_{\text {max }}\right\}$, 
and the CA as the area where the probability of cooperation $P_{\text {coop }}$ is at least of $p$ :

$\mathrm{CA}=$

$=\left\{\right.$ user locations $m$ such that $\left.\mathcal{P}\left\{\tau_{\max , m} \leq \tau_{\max }\right\} \geq p\right\}$.

For instance, let us assume that the maximum delay allowed by the network within the cluster of Fig. 1 to perform BS cooperation is 8 chip periods. Therefore, the CA may be defined as the area within the cluster where the maximum delay of any composite channel is such that $\tau_{\max , m} \leq 8 T_{c}$ with a probability of at least $95 \%$. From Fig. 3, it can be stated that the curve located at the far right that fulfills $\mathcal{P}\left\{\tau_{\max , m} \leq 8 T_{c}\right\} \geq$ 0.95 is the one labeled with $N_{\text {coop }}=4$. Therefore, only those users whose maximum ToAD in (29) is equal or less than 4 chip periods, that is, $\Delta T_{m k} \leq N_{\text {coop }} T_{c}$, with $N_{\text {coop }}=4$, will be served through joint processing. From this example, it is straightforward to see that the CA can be defined by specifying the maximum delay threshold $\tau_{\max }$, or equivalently, the $N_{\text {coop }}$ integer value, since both values are related through Fig. 3 .

From a practical point of view, a user $m$ would need to feed back to its serving BS only the values of the ToA of the rest of BSs in the cluster, $\tau_{m, k, 1}$, for $k=$ $2, \ldots, K$, in addition to local CSI $\mathbf{h}_{m, 1, l}, \forall l$. The serving BS would calculate the $\Delta T_{m k}$ values, for $k=2, \ldots, K$, and depending on the established $N_{\text {coop }}$ value, would activate or not, the joint processing for that user.

\subsection{Statistical modeling of cross-correlation between} codewords

As stated before, the first term (27) of the average MUI power is the multi-user interference arriving from the first path $(l=1)$ of the BSs different from the one whose RAKE finger is synchronized to. Then, the average MUI power can be expressed in terms of relative ToADs as:

$$
\begin{aligned}
& P_{m}^{\mathrm{MUI}}=\sum_{q=1}^{K} \sum_{\substack{i=1 \\
i \neq m}}^{M}\left[\sum_{\substack{k=1 \\
k \neq q}}^{K} P_{i, k} \mathbf{w}_{i, k}^{H} E\left[\mathbf{h}_{m, k, 1}^{H} \mathbf{h}_{m, k, 1}\right] .\right. \\
& \mathbf{w}_{i, k} \rho_{m i}^{2}\left(\Delta T_{m q k}\right)+ \\
& \sum_{k=1}^{K} P_{i, k} \sum_{l=2}^{L} \mathbf{w}_{i, k}^{H} E\left[\mathbf{h}_{m, k, l}^{H} \mathbf{h}_{m, k, l}\right] . \\
& \left.\mathbf{w}_{i, k} \rho_{m i}^{2}\left(\tau_{m, q, 1}-\tau_{m, k, l}\right)\right],
\end{aligned}
$$

Table 1 ToADs $\Delta T_{m q k}$ associated to the cases defined in (37) for a particular cluster of $K=3 \mathrm{BSs}$

\begin{tabular}{l||l|l|l}
\hline \multicolumn{1}{c||}{$\mathrm{q}=1$} & $\mathrm{q}=2$ & $\mathrm{q}=3$ \\
\hline \hline $\mathrm{k}=1$ & NO MUI & $\mathrm{C} 1: \Delta T_{m 21}$ & $\mathrm{C} 1: \Delta T_{m 31}$ \\
\hline $\mathrm{k}=2$ & $\mathrm{C} 2: \Delta T_{m 12}$ & NO MUI & C3: $\Delta T_{m 32}$ \\
\hline $\mathrm{k}=3$ & $\mathrm{C} 2: \Delta T_{m 13}$ & $\mathrm{C} 3: \Delta T_{m 23}$ & NO MUI \\
\hline
\end{tabular}

where

$\Delta T_{m q k}=\tau_{m, q, 1}-\tau_{m, k, 1}, \quad q, k=1, \ldots, K, k \neq q$,

is the relative ToAD between the first paths from the $q$ th and the $k$ th BSs. Notice that the correlative numbering of the BSs for each user allows for a common characterization of the relative ToADs, since $\tau_{m, 1,1}$ is always the ToA of the first path of the composite channel, $\tau_{m, 2,1}$ is always the ToA of the second BS channel arriving to the user (BS2) and so on.

The aim of the paper is to characterize and mitigate the average MUI power in (34)-(35) experienced by the users in the cluster, but taking into account that only local CSI is available at each BS and assuming that a limited amount of additional feedback can be used for sending the $\tau_{m, k, 1}$, for $k=2, \ldots, K$, values. Following the assumption A.2., the mean gains $E\left[\mathbf{h}_{m, k, 1}^{H} \mathbf{h}_{m, k, 1}\right]$ in (34) are supposed to be higher than those in (35). Due to this reason, we start our analysis of the MUI taking into account only term (34) in the following.

Considering the cross-correlation term of (34), we can distinguish three different cases:

$$
\begin{aligned}
& \text { case C1 }: \rho_{m i}^{2}\left(\Delta T_{m 1 k}\right), k=2, \ldots, K, \\
& \text { case C2 }: \rho_{m i}^{2}\left(\Delta T_{m q 1}\right), q=2, \ldots, K, \\
& \text { case C3 }: \rho_{m i}^{2}\left(\Delta T_{m q k}\right)=\rho_{m i}^{2}\left(\Delta T_{m q 1}-\Delta T_{m 1 k}\right), \\
& \quad q, k=2, \ldots, K \text { and } k \neq q .
\end{aligned}
$$

Notice that for all cases in (37), the relative ToAD is expressed in terms of the ToAD defined in (29), that is, $\Delta T_{m q 1}$ in (36) is equal to $\Delta T_{m q}$ in (29). In Section 3.1, the $\mathrm{CA}$ has been defined based on the statistical distribution of the ToADs. Following the same approach, the expected cross-correlation values of (37) can be derived. Cases C1 and C2 consider ToADs between any BS and BS1, whereas case C3 includes the ToADs between any BS excluding BS1. Table 1 shows the ToADs involved in each case of (37) for $K=3$.

Assuming that all possible user locations are uniformly distributed over the CA, the ToADs for cases $\mathrm{C} 1, \Delta T_{m 1 k}$, and $\mathrm{C} 2, \Delta T_{m q 1}$, can be shown to be uniformly distributed within a range of $N_{\text {coop }} T_{c}$ seconds. 
However, case C3 in (37) depends on the relative ToAD between two BSs different from BS1, and it is formed by the sum of two uniformly distributed independent random variables, $\Delta T_{m q 1}$ and $\Delta T_{m 1 k}$, with $q \neq k$. Therefore, the statistical distribution of the corresponding ToADs for the different cases described above is given by:

$$
\begin{aligned}
\text { case C1 } & : f_{\Delta T_{m 1 k}}\left(\Delta T_{m 1 k}\right)=\frac{1}{N_{\text {coop }} T_{c}}, \\
& -N_{\text {coop }} T_{c}, \leq \Delta T_{m 1 k}<0, k=2, \ldots, K
\end{aligned}
$$

case C2: $f_{\Delta T_{m q 1}}\left(\Delta T_{m q 1}\right)=\frac{1}{N_{\text {coop }} T_{c}}$

$$
0<\Delta T_{m q 1} \leq N_{\text {coop }} T_{c}, q=2, \ldots, K,
$$

case C3 $f_{\Delta T_{m q k}}\left(\Delta T_{m q k}\right)=$

$$
\begin{aligned}
& f_{\Delta T_{m q 1}}\left(\Delta T_{m q 1}\right) * f_{\Delta T_{m 1 k}}\left(\Delta T_{m 1 k}\right), \\
& -N_{\text {coop }} T_{c} \leq \Delta T_{m q k} \leq N_{\text {coop }} T_{c}, \\
& q, k=2, \ldots, K, k \neq q
\end{aligned}
$$

where $f_{X}(x)$ stands for the Probability Density Function $(\mathrm{PDF})$ of random variable $X$ and $*$ is the convolution operator. Note that throughout this process, $N_{\text {coop }}$ is a parameter known at the cluster level, since its value has been established during the cooperation area definition procedure.

Once the statistical distributions of the relative delays $\Delta T_{m q k}$ have been derived, it is possible to characterize the cross-correlation terms of (37) from a statistical point of view. Looking at the expression of the average MUI power given in (34), the expectation operator was not then applied to the cross-correlation $\rho_{m i}^{2}\left(\Delta T_{m q k}\right)$ since the time difference $\Delta T_{m q k}$ was assumed to be known (deterministic). Now that a statistical model of $\Delta T_{m q k}$ has been found for a uniform distribution of users in the cluster, the cross-correlation term $\rho_{m i}^{2}(\cdot)$ has to be considered a random variable.

We have modeled the random variable $\rho_{m i}^{2}\left(\Delta T_{m q k}\right)$ performing Monte-Carlo simulations: for each possible pair of users locations $m$ and $i$, and for each possible code of spreading factor $N$, the statistical distributions from (38) are generated and the Mean Squared Value (MSV) of each cross-correlation term defined as

$$
\begin{aligned}
& \text { case } \mathrm{C} 1: \xi_{m i, \mathrm{C} 1}^{2}=E\left[\rho_{m i}^{2}\left(\Delta T_{m 1 k}\right)\right], k=2, \ldots, K, \\
& \text { case } \mathrm{C} 2: \xi_{m i, \mathrm{C} 2}^{2}=E\left[\rho_{m i}^{2}\left(\Delta T_{m q 1}\right)\right], q=2, \ldots, K, \\
& \text { case C3: } \xi_{m i, \mathrm{C} 3}^{2}=E\left[\rho_{m i}^{2}\left(\Delta T_{m q k}\right)\right], \\
& \quad q, k=2, \ldots, K, k \neq q,
\end{aligned}
$$

is evaluated. Note that the MSV is the parameter which directly influences the average MUI power from (34).
Within a cluster of $K \mathrm{BSs}$, there are $(K-1)$ random variables $\Delta T_{m 1 k},(K-1)$ random variables $\Delta T_{m q 1}$, and the remaining are expressed as $\Delta T_{m q k}, q, k=2, \ldots, K$, $k \neq q$ (see example of Table 1 ). Therefore, it is possible to estimate the total contribution of the crosscorrelation terms in (34) to the average MUI power by:

$\xi_{m i}^{2}=(K-1) \xi_{m i, \mathrm{C} 1}^{2}+(K-1) \xi_{m i, \mathrm{C} 2}^{2}+(K-2)(K-1) \xi_{m i, \mathrm{C} 3}^{2}$.

Note that up to this point, the values of $P_{i, k}, \mathbf{w}_{i, k}$ and $\mathbf{h}_{m, k, 1}$ have not been considered. In our framework, we assume that the design of downlink beamformers and powers, and the design of the scheme that will tackle the contribution of cross-correlation terms from (40) are carried out in different time scales. Basically, downlink beamformers and powers can be designed with local CSI available at each BS, whereas the scheme dealing with (40) requires the feedback of $\tau_{m, k, 1}, k=2, \ldots, K$, from the user, and in this paper, it is considered as a static solution for a given cooperation area. More details are given in the next Section.

Considering now the contribution of term (35) to the average MUI power, a similar statistical characterization of the difference of delays $\left(\tau_{m, q, 1}-\tau_{m, k, l}\right)$, as in (38) for $\Delta T_{m q k}$, could be obtained. Expressing the difference of delays as $\left(\tau_{m, q, 1}-\tau_{m, k, l}\right)=\Delta T_{m q k}+\left(\tau_{m, k, 1}-\tau_{m, k, l}\right)$, it can be noted that the statistical model will depend on the distribution of $\Delta T_{m q k}$, which is known, and on the distributions of the delays for each channel with respect to the delay of its first path, $\left(\tau_{m, k, 1}-\tau_{m, k, l}\right)$. This last term depends on the particular CSI and not on the user location. Therefore, any scheme proposed to mitigate the average MUI power contribution in (35) would require knowledge of timely and accurate CSI, which seems rather unrealistic under limited feedback requirements. Consequently, only contributions from (34) to the average MUI power are included in our model.

Regarding the expression obtained in (40), the question now is how the allocation of the spreading codes to the users could mitigate the received average MUI power. Clearly, the code allocation should aim to minimize the parameter $\xi_{m i}^{2}$.

\section{Spreading Code Allocation Scheme based on minimum correlation}

Code allocation schemes for WCDMA systems have been thoroughly studied in the literature due to the problem of code blocking and the constraint on the number of available orthogonal sequences, which is limited by the value of the spreading factor. Regarding 
the code blocking problem, static and dynamic schemes have been proposed to minimize the number of code reassignments [51-53]. To avoid the capacity restriction in the number of available spreading codes, one possible approach is to employ additional non-orthogonal spreading codes. In this case, optimum spreading code sets have been identified as the ones that minimize cross-correlation values. Furthermore, some approaches iteratively update each user spreading code according to MUI conditions [54-56].

In this paper, we exploit the idea of minimum crosscorrelation values from a different point of view. The average MUI power expression given by (34) points out the influence of the code correlation values on the interference level of the cluster. In fact, the MSV of cross-correlation terms in (40) can be seen as an average of the MUI influence on the user when a certain spreading code is allocated by the set of cooperating BSs. Hence, the key idea of mitigating the average MUI power level of the system using an optimized assignment of the available OVSF codes ${ }^{7}$ arises. In this paper, a multi-base spreading code allocation scheme, denoted by Minimum Correlation (MC), is proposed for systems with BS cooperation. Specifically, spreading codes are allocated in such a way that minimum average crosscorrelation between active users can be achieved.

Let us assume that the average MUI power experienced by the $m$ th user depends on the sum of different cross-correlation terms as stated in (40). In a given layer of the OVSF code tree, there are $N$ available spreading codes. For each possible pair of these codes and a particular value of $N_{\text {coop }}$ or cooperation area, $\xi_{m i}^{2}$ can be pre-estimated through Monte-Carlo simulations as derived in the previous Section. Based on these values, the MC scheme allocates codes to new users on a minimum average cross-correlation basis, mitigating the MUI power present in the cluster. The steps will be the following:

1. Build matrix $\boldsymbol{\Xi}$ of size $[N \times N]$ as follows:

$$
\boldsymbol{\Xi}=\left(\begin{array}{cccc}
0 & \xi_{12}^{2} & \cdots & \xi_{1 N}^{2} \\
\xi_{21}^{2} & 0 & \cdots & \xi_{2 N}^{2} \\
\vdots & \vdots & \ddots & \vdots \\
\xi_{N 1}^{2} & \xi_{N 2}^{2} & \cdots & 0
\end{array}\right)
$$

2. Assume that spreading code \#1 is the only one assigned (usually used for pilot and control channel) and a new user admission is requested to the cluster. In this case, the MC scheme allocates the spreading code $j$ for the new user such that $\xi_{1 j}^{2}$ is minimum.

\footnotetext{
7 OVSF codes have been considered throughout the paper,
} but average MUI contribution could be calculated as stated in the previous Section for any set of channelization codes.
3. When a new user enters the cluster, spreading code $j$ that minimizes the sum of cross-correlation terms due to active users is chosen as:

$$
j=\arg \min \left\{\sum_{i \text { active }} \xi_{j i}^{2}\right\} .
$$

In practical WCDMA based systems, spreading codes from different OVSF layers are needed, since users may require services with different data rates. In this case, the $\mathrm{MC}$ assignment scheme can be modified as follows: for a given layer, matrix $\boldsymbol{\Xi}$ is built taking only into account the set of non-blocked spreading codes.

In this paper, the MC scheme is used as a static allocation policy, that is, given a certain channel distribution, MSVs $\xi_{m i}^{2}$ are pre-calculated following the statistical model proposed in Section 3.2, and stored in an off-line table. Therefore, the MC scheme allows a distributed implementation, since the off-line tables, including the spreading code ordering, can be locally stored at each BS and the amount of inter-base information exchange is decreased. However, this scheme can also be dynamically updated using current channel gain estimates and PDP, depending on the computational capacity of the BSs involved and the available feedback bits. In this case, in order to obtain a distributed implementation of the MC assignment scheme, it is possible to follow a decentralized approach, as the one proposed in [57] or an iterative approach [58], where matrix $\boldsymbol{\Xi}$ is iteratively updated by each BS.

To summarize, Algorithm 1 states the main off-line steps that need to be performed at the cluster side for all possible $N_{\text {coop }}$ values according to the cluster characteristics (i.e., cell radius) whereas Algorithm 2 summarizes the actions required for each new user entering the cluster. Note that the output of Algorithm 1 is a given spreading code ordering for each possible value of $N_{\text {coop }}$, and that the definition of the $\tau_{\max }$ value provided by the network is out of the scope of this paper.

\section{Simulation Results}

We consider a static cluster with $K=3$ BSs (see Fig. 1), each one equipped with an array of $N_{a}=3$ or $N_{a}=2$ antennas, and single-antenna users. WCDMA signals with a spreading factor of $N_{1}=16$ or $N_{2}=32$ are used and the per-BS power constraint is set to $43 \mathrm{dBm}$. The initial position of each user is uniformly distributed over the cluster area. Without loss of generality, each user is assigned to the BS whose $\tau_{m, k, 1}$ is the minimum, i.e., BS1, for CSI feedback purposes. 

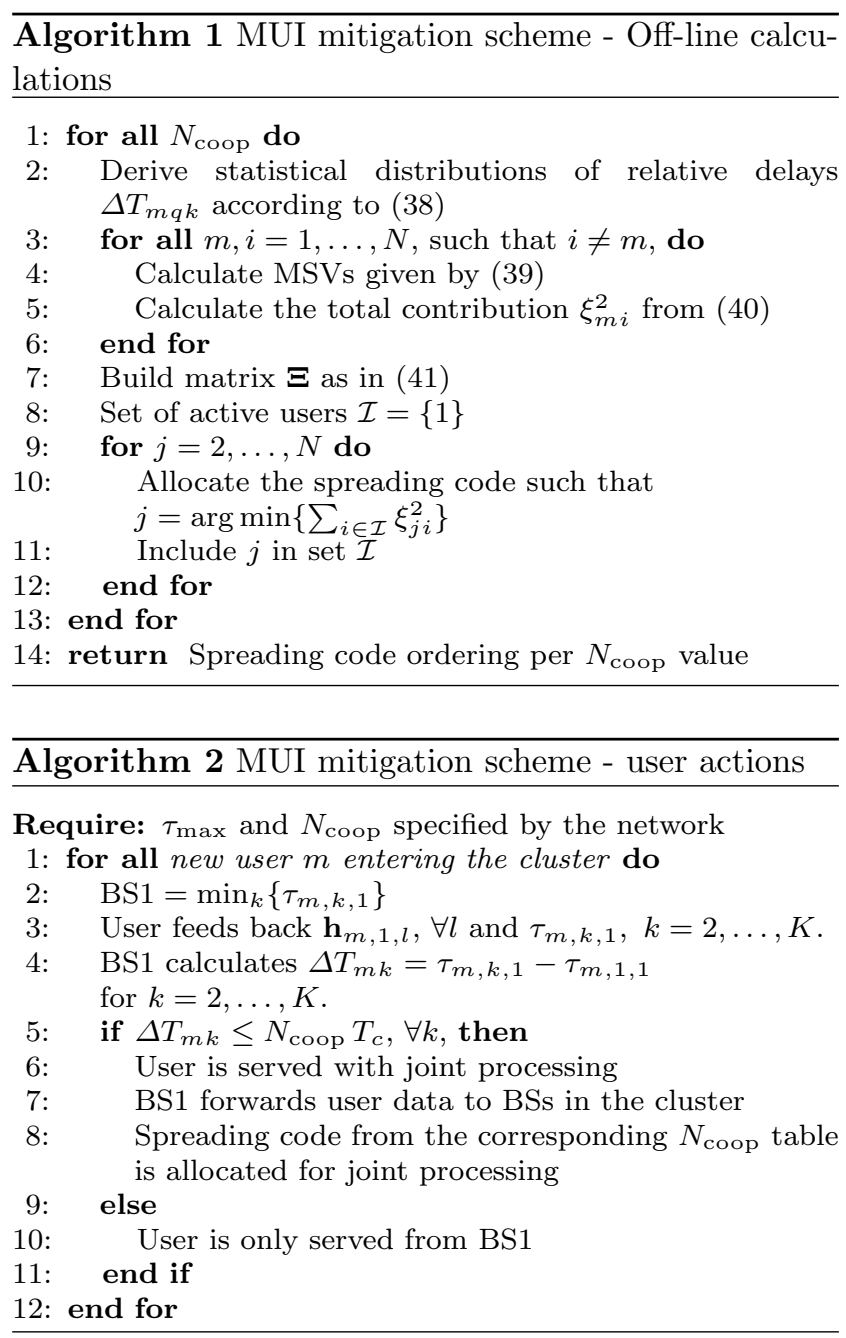

The channel $\mathbf{h}_{m, k, l}, \forall l$, between each user $m$ and each BS $k$ is modeled using the Spatial Channel Model (SCM) for MIMO systems proposed by the 3GPP [40]. Assuming a urban micro-cell environment, with $1 \mathrm{Km}$ distance between BSs, the PDP for each BS-user channel has been independently generated and consists of 6 distinct paths $\tau_{m, k, l} \sim \mathcal{U}[0,1.2 \mu s]$. The powers for each path are exponentially decaying in time, with the addition of lognormal randomness independent of the path delay.

Base station cooperation within the cluster is performed at the physical layer by means of the JPCOBVUL (Joint Power Control and Beamforming - Virtual UpLink) algorithm of [38] and [39]. This algorithm is an extension of reference [59] for systems with BS cooperation: in a first step, per-BS transmit beamformers $\mathbf{w}_{i, k}$ are obtained from a simpler virtual uplink formulation, and in a second step, downlink transmit powers $P_{i, k}$ are jointly updated from a set of linear equations. Nevertheless, some matrices from [38] have been redefined in order to include the modified MUI term.

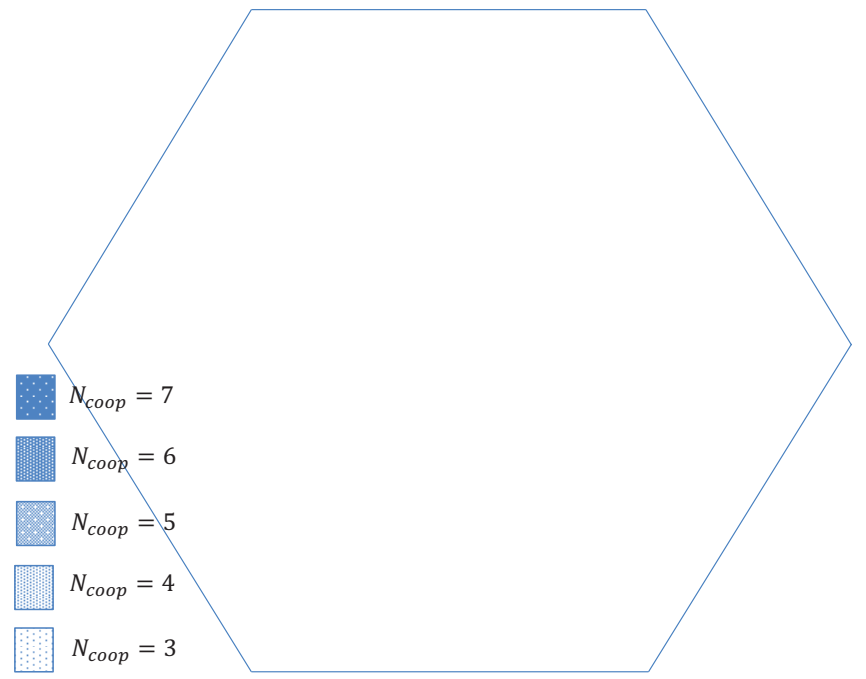

Fig. 4 Cooperation areas for a cluster with $K=3 \mathrm{BSs}$ and $3 \leq N_{\text {coop }} \leq 7$

In the JPCOB-VUL algorithm, the QoS constraint is represented by a certain SINR requirement, which is common to all the users in the cluster. For a given SINR constraint, the JPCOB-VUL algorithm is simulated over independent channel realizations and distributions of users. In each run, users have to achieve the required SINR within a maximum of 15 iterations. Hence, the algorithm fails when, in a given run, at least one user does not achieve the required SINR.

\subsection{Cooperation area}

Applying the approximation from (29) for each possible location in the cluster area, we find that the maximum $\Delta T_{m k}$ for this scenario is $10 T_{c}$, where $T_{c}=1 / 3.84$. $10^{-6} \mathrm{~s}$. Therefore, the set of ToADs follow a uniform distribution according to $\Delta T_{m k} \sim \mathcal{U}\left[0,10 T_{c}\right]$. In order to define the $\mathrm{CA}$, we need to state the value for the predefined threshold $\tau_{\max }$. As in the example stated before, we assume that the maximum delay allowed by the cluster is $\tau_{\max }=8 T_{c}$ with a probability of at least $95 \%$ of the time. As before, from Fig. 3 we see that the only curve fulfilling this requirement is the one labeled with $N_{\text {coop }}=4$, meaning that the CA is comprised of the cluster locations where $\mathcal{P}\left\{\tau_{\max , m} \leq 8 T_{c}\right\} \geq 0.95$, which corresponds to the cluster locations labeled with $N_{\text {coop }}=4$ in Fig. 4 .

Once the $N_{\text {coop }}$ value is stated, BSs in the cluster resort to the corresponding spreading code ordering for the users entering the cluster. 


\subsection{Probability of Admission}

In this Section, we evaluate the probability of admitting users under two spreading code allocation schemes, the $\mathrm{MC}$ and the natural ${ }^{8}$ or unsorted code allocation scheme. The static cluster from Fig. 1 with $N_{a}=2$ or $N_{a}=3$ antenna arrays in each BS is considered. Spreading factors of $N_{1}=16$ and $N_{2}=32$ are simulated, which imply SINR constraints of 4 and $2 \mathrm{~dB}$ after despreading, respectively.

In order to evaluate the probability of admission, each run of the JPCOB-VUL algorithm is initialized with $M=1$ active user. Once this user achieves the SINR requirement, a new user enters the cluster. If the JPCOB-VUL algorithm fulfills the SINR requirement for all the users, included the last one, simulation goes on adding users until the maximum capacity, $M=N$. In any other case, simulation stops. This procedure is repeated for 1500 independent channel realizations and distributions of users in the case of $N_{1}=16$ and for 750 in the $N_{2}=32$ case. Therefore, for a given number of active users, the probability of admission is here defined as the number of runs in which at least that number of users achieve the required SINR, divided by the total number of independent runs (this is a slight modification of the CDF).

Fig. 5 and Fig. 6 show the estimated CDF of the probability of admission of MC and Natural schemes for a cooperation cluster of $K=3 \mathrm{BSs}$ and $N_{1}=16$ and $N_{2}=32$ spreading factors, respectively. It can be observed in both figures that the MC scheme outperforms the Natural scheme when the same number of antennas per BS is considered, regardless of the spreading factor. This improvement is also noticed when comparing the different configurations showed in each figure. For example, in Fig. 6, there is a $75 \%$ increase in the probability of admission when using the MC scheme with $N_{a}=2$ with respect to the corresponding Natural case for a medium traffic load ( $M=16$ active users). The same comparison over Fig. 5 yields a $90 \%$ increase of the probability of admission for $M=8$ active users.

Comparing now both Fig. 5 and Fig. 6, it can be seen that improving the beamforming capacity of each BSs, that is, increasing the number of antennas in each array from $N_{a}=2$ to $N_{a}=3$, allows a general enhancement of the probability of admission for medium and high traffic loads. For example, in Fig. 6, the Natural scheme with $N_{a}=3$ outperforms the MC scheme with $N_{a}=2$. However, for low spreading factors and hence, high data rates, optimizing the spreading code

8 OVSF codes are sequentially assigned from 1 to $N$ through the OVSF layer.

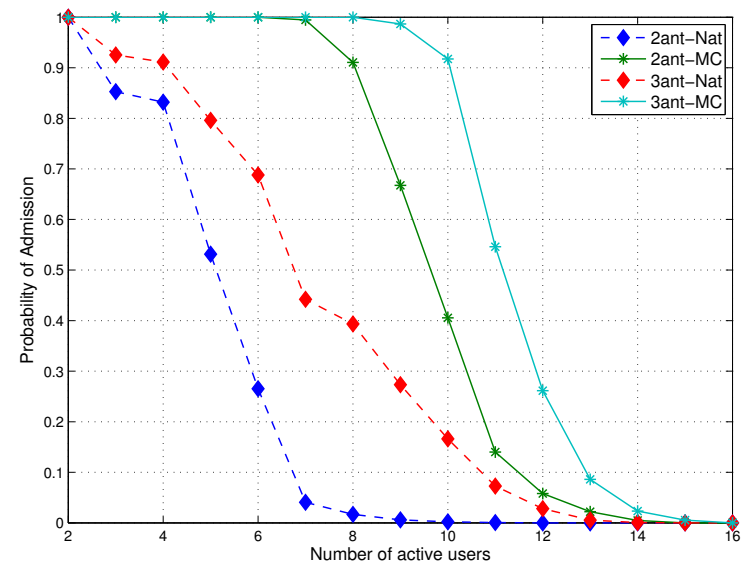

Fig. 5 Estimated CDF of the probability of admission for a cluster with $K=3 \mathrm{BSs}, N_{a}=2$ or $N_{a}=3$ antenna arrays, and a SINR requirement of $4 \mathrm{~dB}$. Results are shown for the Natural and MC schemes, and $N_{1}=16$

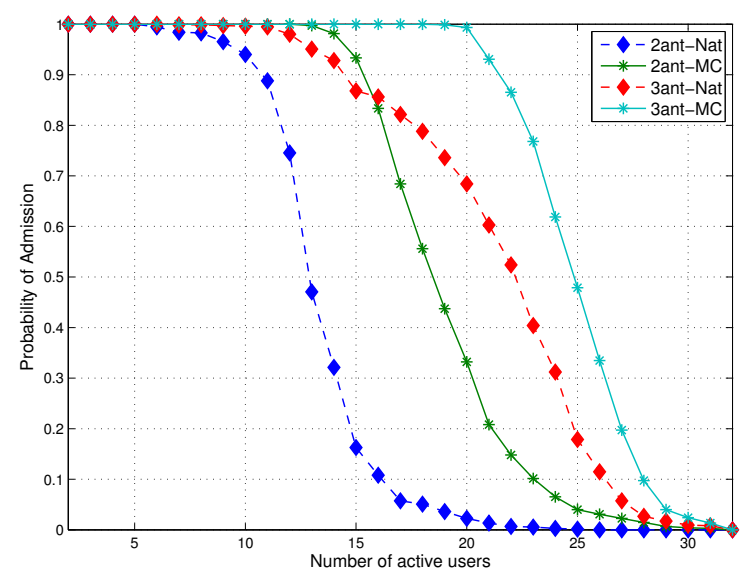

Fig. 6 Estimated CDF of the probability of admission for a cluster with $K=3 \mathrm{BSs}, N_{a}=2$ or $N_{a}=3$ antenna arrays, and a SINR requirement of $2 \mathrm{~dB}$. Results are shown for the Natural and MC schemes, and $N_{2}=32$

allocation as sown in Fig. 5 can bring higher gains than increasing the beamforming capability of the BSs.

\section{Conclusion}

In this paper, a framework for mitigating the multiuser interference in the downlink of a partially asynchronous WCDMA cluster with base station cooperation has been presented. Assuming that local channel state information is available at the base stations, and that a limited amount of additional feedback per user can be used, cooperation areas have been defined in order to mitigate the impact of the time-shifted spreading codes in the average multi-user interference power in 
the cluster. By providing a statistical modeling of the average multi-user interference power experienced by each user in the cluster, a minimum correlation spreading code allocation scheme for users served with joint processing has been derived. Simulation results show that for low spreading factors, optimizing the spreading code allocation brings higher gains than increasing the beamforming capability of the base stations, whereas in medium and high traffic loads, improving the beamforming capability of the base stations can bring a general enhancement of the probability of users' admission. In future work, we plan to adapt this multi-user interference mitigation framework to the scenario of a dense deployment of small cells, where users could benefit of the potential gains of cooperation between base stations.

Acknowledgements C. Botella's work has been partially supported by the Spanish MINECO Grants CONSOLIDERINGENIO 2010 CSD 2008-00010 COMONSENS and RACHEL TEC2013-47141-C4-4-R. G. Piñero and M. de Diego's work has been supported by European Union ERDF and Spanish Government through TEC2012-38142-C04 Project, and Generalitat Valenciana through PROMETEOII/2014/003 Project.

\section{References}

1. V. Jungnickel, K. Manolakis, W. Zirwas, B. Panzner, V. Braun, M. Lossow, M. Sternad, R. Apelfröjd, T. Svensson (2014), The role of small cells, coordinated multipoint, and massive MIMO in 5G, IEEE Communications Magazine, 52(5), 44-50.

2. E. Hossain, Z. Lei (2013), Multicell cooperation, IEEE Wireless Communications, special issue, 20(1).

3. M.K. Karakayali, G.J. Foschini, R.A. Valenzuela (2006), Network coordination for spectrally efficient communications in cellular systems, IEEE Wireless Communications, 13(4), 56-61.

4. Qinghe Du, Xi Zhang (2011), Base-station selection for QoS provisioning over distributed multi-user MIMO links in wireless networks, in Proc. IEEE INFOCOM.

5. 3GPP TR 36.819, 3rd Generation Partnership Project; Technical Specification Group Radio Access Network; Coordinated multi-point operation for LTE physical layer aspects (Release 11), (2011).

6. 3GPP Release 11, Overview of 3GPP Release 11 V0.2.0 (2014).

7. Nokia Solutions and Networks, LTE-Advanced (Release 12 and Beyond), (2014), available at http: //www.networks.nokia.com/index.php?q=system/files/ document/nsn_lte_a_evolution_white_paper_1.pdf

8. F. Rusek, D. Persson, B.K. Lau, E.G. Larsson, T.L. Marzetta, O. Edfors, F. Tufvesson (2013), Scaling up MIMO: opportunities and challenges with very large arrays, IEEE Signal Processing Magazine, 30(1), 40-60.

9. Nokia Solutions and Networks, Looking ahead to 5G, (2013), available at http://networks.nokia.com//file/ 28771/nsn-5g-white-paper

10. Ericsson, HSPA Evolution - beyond 3GPP Release 10, (2011), available at http://www.3g4g.co.uk/Hspa/HSPAE_ WP_1107_Ericsson.pdf
11. Nokia Siemens Networks, Long Term HSPA Evolution meets ITU IMT-Advanced requirements, (2012), available at http://nsn.com/system/files/document/nokia_ siemens_networks_long_term_hspa_evolution_meets_ itu_imt-advanced_requirements_18_04_12_online.pdf

12. Qualcomm Incorporated, HSPA+ Advanced Smart Networks: multipoint transmission, (2011), available at https://www .qualcomm.com/system/files/document/ files/Research_HSPA_Advanced_Smart_Networks_\%C3\% A2\%E2\%82\%AC\%E2\%80\%9C_Multipoint_Transmission.v2 . 20110210.pdf

13. Nokia Solutions and Networks, Taking HSPA to the next level with Release 12 and beyond, (2014), available at http://networks.nokia.com/sites/default/files/ document/nokia_long_term_hspa_evolution_white_ paper_1.pdf

14. 3GPP TSG-RAN, RP-101439, Proposed study item on HSDPA multipoint transmission, (2010).

15. 3GPP TR 25.872, 3rd Generation Partnership Project; Technical Specification Group Radio Access Network; High Speed Packet Access (HSDPA) multipoint transmission (Release 11), (2011).

16. 3GPP TSG-RAN , RP-111375, HSDPA Multiflow data transmission, (2011).

17. 3GPP TSG-RAN WG1, R1-104913, Multi-cell transmission techniques for HSDPA, (2010).

18. 3GPP TSG-RAN WG1, R1-110123, Candidate schemes for multi-point HSDPA, (2011)

19. V. Hytonen, O. Puchko, T. Hohne, T. Chapman (2011), High-speed single-frequency network for HSDPA, in Proc. IEEE Swedish Communication Technologies Workshop.

20. Wei Yang, Yongyu Chang, Shuhui Liu, Dacheng Yang (2011), Efficient multi-point transmission scheme for HSDPA networks, in Proc. IEEE Vehicular Technology Conference (VTC-Fall).

21. D. Petrov, I. Repo, M. Lampinen (2012), Overview of single frequency multipoint transmission concepts for HSDPA and performance evaluation of intra-site multiflow aggregation scheme, in Proc. IEEE Vehicular Technology Conference (VTC-Spring).

22. A. Yaver, P. Marsch, K. Pawlak, F.S. Moya (2012), On the joint usage of MIMO and multiflow in evolved HSPA networks, in Proc. IEEE International Conference on Communications (ICC).

23. Qualcomm Technologies, HSPA+ Multiflow. Solution for cell edge performance improvement and dynamic load balancing, (2014), available at https://www.qualcomm.com/ media/documents/files/hspa-multiflow.pdf

24. Nokia Solutions and Networks, Setting up HSPA+ heterogenous networks for the best customer experience, (2014), available at http://networks.nokia.com/ sites/default/files/document/nsn_setting_up_hspa_ hetnets_white_paper_1.pdf

25. S. Venkatesan (2007), Coordinating base stations for greater uplink spectral efficiency in a cellular network, in Proc. IEEE International Symposium on Personal, Indoor and Mobile Radio Communications (PIMRC).

26. F. Boccardi, H. Huang (2007), Limited downlink network coordination in cellular networks, in Proc. IEEE International Symposium on Personal, Indoor and Mobile Radio Communications (PIMRC).

27. A. Papadogiannis, D. Gesbert, E. Hardouin (2008), A dynamic clustering approach in wireless networks with multicell cooperative processing, in Proc. IEEE International Conference on Communications (ICC). 
28. F. Boccardi, H. Huang, A. Alexiou (2008), Network MIMO with reduced backhaul requirements by MAC coordination, in Proc. IEEE Asilomar Conference on Signals, Systems and Computers.

29. Hongyuan Zhang, N.B. Mehta, A.F. Molisch, Jin Zhang, Huaiyu Dai (2008), Asynchronous interference mitigation in cooperative base station systems, IEEE Transactions on Wireless Communications, 7(1), 155-165.

30. Sen Bong Gee, Zhongding Lei, Yong Huat Chew (2011), Cooperative multiuser MIMO precoding design for asynchronous interference mitigation, in Proc. IEEE Global Telecommunications Conference workshops (GLOBECOM).

31. Y. Medjahdi, M. Terre, D. Le Ruyet, D. Roviras, A. Dziri (2011), Performance analysis of asynchronous OFDM/FBMC based multi-cellular networks, IEEE Transactions on Wireless Communications, 10(8), 2630-2639.

32. B.W. Zarikoff, J.K. Cavers (2010), Coordinated multiCell systems: carrier frequency offset estimation and correction, IEEE Journal on Selected Areas in Communications, 28(9), 1490-1501.

33. O. Awoniyi, N.B. Mehta, L.J. Greenstein (2003), Characterizing the orthogonality factor in WCDMA downlinks, IEEE Transactions on Wireless Communications, 2(4), 621-625.

34. N.B. Mehta, L.J. Greenstein, T.M. Willis, Z. Kostic (2003), Analysis and results for the orthogonality factor in WCDMA downlinks, IEEE Transactions on Wireless Communications, 2(6), 1138-1149.

35. N.B. Mehta, A.F. Molisch, L.J. Greenstein (2006), Macrocell-wide behavior of the orthogonality factor in WCDMA downlinks, IEEE Transactions on Wireless Communications, 5(12), 3394-3399.

36. C. Botella, G. Piñero, A. González, M. de Diego (2006), Spreading sequence assignment in WCDMA for distributed antenna arrays based on interference knowledge, in Proc. IEEE Workshop on Signal Processing Advances in Wireless Communications (SPAWC).

37. Yu-Jung Chang, Zhifeng Tao, Jinyun Zhang, C.-C. Jay Kuo (2008), A graph-based approach to multi-cell OFDMA downlink resource allocation, Proc. IEEE Global Telecommunications Conference (GLOBECOM).

38. G. Piñero, C. Botella, A. González, M. de Diego, N. Cardona (2004), Downlink power control and beamforming for a cooperative wireless system, Proc. IEEE International Symposium on Personal, Indoor and Mobile Radio Communications (PIMRC).

39. C. Botella, G. Piñero, A. González, M. de Diego (2008), Coordination in a multi-cell multi-antenna multi-user WCDMA system: a beamforming approach, IEEE Transactions on Wireless Communications, 7(11), 4479-4485.

40. 3GPP TR 25.996, 3rd Generation Partnership Project; Technical Specification Group Radio Access Network; Spatial channel model for Multiple Input Multiple Output (MIMO) simulations (Release 11), (2012).

41. K. Cheun (1997), Performance of direct-sequence spreadspectrum RAKE receivers with random spreading sequences, IEEE Transactions on Communications, 45(9), $1130-1143$.

42. P. K. Gkonis, G. V. Tsoulos, D. I. Kaklamani (2011), Performance evaluation of MIMO-WCDMA cellular networks in multiuser frequency selective fading environments, Wireless Communications and Mobile Computing, 13(1), 72-84.

43. G.E. Bottomley, T. Ottosson, Y.-P.E. Wang (2000), A generalized RAKE receiver for interference suppression,
IEEE Journal on Selected Areas in Communications, 18(8), 1536-1545.

44. T.L. Fulghum, D. Cairns, C. Cozzo, Y.-P.E. Wang, G.E. Bottomley (2009), Adaptive generalized rake reception in DS-CDMA systems, IEEE Transactions on Wireless Communications, 8(7), 3464-3474.

45. C.-X. Wang, X. Hong, H. Wu, W. Xu (2007), Spatialtemporal correlation properties of the $3 \mathrm{GPP}$ spatial channel model and the Kronecker MIMO channel model, EURASIP Journal on Wireless Communications and Networking, Article ID 39871.

46. Jingya Li, T. Svensson, C. Botella, T. Eriksson, Xiaodong $\mathrm{Xu}$, Xin Chen (2011), Joint scheduling and power control in coordinated multi-point clusters, in Proc. IEEE Vehicular Technology Conference (VTC-fall).

47. C. Botella, T. Svensson, Xiaodong Xu, Zhang Hui (2010), On the performance of joint processing schemes over the cluster area, in Proc. IEEE Vehicular Technology Conference (VTC-spring).

48. A. Papadogiannis, H.J. Bang, D. Gesbert, E. Hardouin (2008), Downlink overhead reduction for multi-cell cooperative processing enabled wireless networks, in Proc. IEEE International Symposium on Personal, Indoor and Mobile Radio Communications (PIMRC).

49. W. Mennerich, W. Zirwas (2010), Implementation issues of the partial CoMP concept, in Proc. IEEE International Symposium on Personal, Indoor and Mobile Radio Communications (PIMRC).

50. 3GPP TSG-RAN WG1, R1-092657, Impact of propagation attenuations and delays of CoMP composite, (2009).

51. Thit Minn, Kai-Yeung Siu (2000), Dynamic assignment of orthogonal variable-spreading-factor codes in WCDMA, IEEE Journal on Selected Areas in Communications, 18(8), 1429-1440.

52. Yu-Chee Tseng, Chih-Minn Chao (2002), Code placement and replacement strategies for wideband CDMA OVSF code tree management, IEEE Transactions on Mobile Computing, 1(4), 293-302.

53. M. Dell'Amico, M.L. Merani, F. Maffioli (2004), A tree partitioning dynamic policy for OVSF codes assignment in wideband CDMA, IEEE Transactions on Wireless Communications, 3(4), 1013-1017.

54. S. Ulukus, R.D. Yates (2001), Iterative construction of optimum signature sequence sets in synchronous CDMA sytems, IEEE Transactions on Information Theory, 47(5), 1989-1998.

55. Jiunn-Tsair Chen, C. Papadias, G.J. Foschini (2004), Space-time dynamic signature assignment for the reverse link of DS-CDMA systems, IEEE Transactions on Communications, 52(1), 120-129.

56. Li Gao, Tan F. Wong (2004), Power control and spreading sequence allocation in a CDMA forward link, IEEE Transactions on Information Theory, 50(1), 105-124.

57. A. Papadogiannis, E. Hardouin, D. Gesbert (2008), A framework for decentralising multi-cell cooperative processing on the downlink, in Proc. IEEE Global Telecommunications Conference (GLOBECOM).

58. H. Skjevling, D. Gesbert, A. Hjørungnes (2008), Low-complexity distributed multibase transmission and scheduling, EURASIP Journal on Advances in Signal Processing, Article ID 741593.

59. F. Rashid-Farrokhi, K.J. Ray Liu, L. Tassiulas (1998), Transmit beamforming and power control for cellular wireless systems, IEEE Journal on Selected Areas in Communications, 16(8), 1437-1450. 